\title{
Giant Enhancement in UV Response of ZnO Nanobelts by Polymer Surface-Functionalization
}

\author{
Chang Shi Lao, ${ }^{\dagger}$ Myung-Chul Park, ${ }^{\ddagger}$ Qin Kuang, ${ }^{\dagger}$ Yulin Deng, ${ }^{\ddagger}$ Ashok K. Sood,, Dennis L. Polla, ${ }^{\#}$ and \\ Zhong L. Wang ${ }^{*}$, \\ School of Materials Science and Engineering, Georgia Institute of Technology, Atlanta, Georgia 30332-0245, \\ School of Chemical and Biomolecular Engineering, Georgia Institute of Technology, Atlanta, Georgia 30332-0620, \\ Magnolia Optical Technologies, Inc., 52-B Cummings Park, Suite 314, Woburn, Massachusetts 01801, and Defense \\ Advanced Research Projects Agency/Microsystems Technology Office (DARPA/MTO), 3701 North Fairfax Drive, \\ Arlington, Virginia 22203-1714 \\ Received July 13, 2007; E-mail: zhong.wang@mse.gatech.edu
}

\begin{abstract}
Zinc oxide $(\mathrm{ZnO})$ is one of the most important nanomaterials for nano-optoelectronics, ${ }^{1}$ sensors, ${ }^{2}$ transistors, ${ }^{3,4}$ and nanopiezotronics. ${ }^{5,6}$ Because of the unique piezoelectric and semiconducting dual properties, $\mathrm{ZnO}$ nanowires (NWs) and nanobelts (NBs) are the fundamental material for nanogenerators, ${ }^{7-9}$ which convert mechanical energy into electricity. With a band gap of $\sim 3.4 \mathrm{eV}$ and an exciton binding energy of $60 \mathrm{meV}, \mathrm{ZnO}$ has huge promise for optical applications, such as UV detection. ${ }^{10}$ However, because of the presence of point defects and confined dimensionality, the UV sensitivity of $\mathrm{ZnO}$ NWs and NBs is limited.
\end{abstract}

In this paper, we demonstrate that the UV response of a $\mathrm{ZnO}$ NB based sensor has been enhanced by close to 5 orders of magnitude after functionalizing its surface with a polymer that has a high UV absorption ability. This giant enhancement in photoconductance is attributed to the energy levels introduced by the polymer lying in the corresponding band gap and in the conduction band of $\mathrm{ZnO}$, which served as a "hopping" state and increased the excitation probability of an electron to the conduction band. This exciting discovery will greatly impact the applications of $\mathrm{ZnO} \mathrm{NWs} / \mathrm{NBs}$ for UV detection.

The device fabrication was achieved using e-beam lithography and focused ion beam (FIB) microscopy. ${ }^{11}$ Surface functionalization is one of the widely used methods to enhance the electric transport properties of different 1D nanostructures. ${ }^{12}$ Because of the special physical and chemical properties of the attached chemical species, devices based on $\mathrm{ZnO}$ NWs/NBs demonstrate unique and interesting properties. ${ }^{13}$ In this paper, a layer-by-layer self-assembly method was used for manipulating and functionalizing polymers onto the surface of $\mathrm{ZnO} N B s .{ }^{14}$ In general, since the functional polymers used for UV sensing was negatively charged, a positively charged polymer, poly(diallyldimethylammonium chloride) (PDADMAC), was chosen to assist the electrostatic assembly of the negatively charged polymer. Surface charge of $\mathrm{ZnO} \mathrm{NBs}$ is negative in aquatic solution according to $\zeta$-potential measurement. Prior to surface coating, the $\mathrm{ZnO} \mathrm{NBs}$ used in the devices were cleaned with oxygen plasma to remove surface chemical residuals and other contaminants before testing or polymer functionalization. For a bare $\mathrm{ZnO} \mathrm{NB}$, PDADMAC was first adsorbed onto its surface by electrostatic attraction force between the negative surface charges of $\mathrm{ZnO}$ and positive charges of PDADMAC. Then, the $\mathrm{ZnO}$ NB was immersed in an anionically charged polymer solution of choice, which was adsorbed on the $\mathrm{ZnO} \mathrm{NB}$ owing to the electrostatic force from the cationically charged PDADMAC molecules.

Different kinds of polymers with different UV absorption abilities and wavelengths were used in the experiment to test their effects

† School of Materials Science and Engineering, Georgia Institute of Technology.

$¥$ School of Chemical and Biomolecular Engineering, Georgia Institute of

Technology.

$\S$ Magnolia Optical Technologies, Inc.

\# DARPA/MTO. on enhancing UV response of the $\mathrm{ZnO} \mathrm{NB}$, including polystyrene sulfate (PSS), poly(styrene-co-maleic acid) (PS-co-MAc), anionically charged poly( $N$-isopropylacrylamide) (PNIPAM), and carboxymethylcellulose (CMC). For the layered coating, these polymers are all negatively charged or modified to possess negative charges. A scanning electron microscopy (SEM) image of these functionalized $\mathrm{ZnO} \mathrm{NBs}$ is shown in the inset of Figure 1b. The blur edge of NBs in the figure indicates successful coating of polymers onto the NBs.

Ultraviolet characteristics of the polymer functionalized $\mathrm{ZnO} \mathrm{NB}$ base devices were tested to investigate their electric transport under UV illumination. Figure 1a is the photoconductance response of the single NB devices fabricated using $\mathrm{ZnO}$ NBs functionalized with different polymers, and the data are plotted for the ratio of the conductance measured when the UV was on $(G)$ and off $\left(G_{0}\right)$. The UV source used in the experiment had a wavelength distribution around $280 \mathrm{~nm}$. The conductance of uncoated $\mathrm{ZnO}$ NB devices (green line) increased for 2.8 times upon UV illumination. The conductance of the PS-co-MAc-coated NB (dark line) increased for 50 times upon UV illumination. The conductance for PNIPAM and CMC-coated ZnO NBs was increased by 100 and 15 times, respectively (see Supporting Information Figure S1). Surprisingly, the photoconductance of the PSS-coated $\mathrm{ZnO} \mathrm{NB}$ (red line) increases by 75000 times when UV was on. This is a sharp contrast to the other three polymers-coated $\mathrm{ZnO}$ NBs. It should be noted that dry PSS polymer itself is nonconductive and showed almost no response to UV illumination (blue line), thus, on its own it cannot contribute to the huge increase in conductance. In contrast, PSScoated $\mathrm{ZnO} \mathrm{NB}$ showed a much smaller response $(\sim 2)$ to the light of 523 and $680 \mathrm{~nm}$ wavelength (see Supporting Information Figure $\mathrm{S} 2$ ). This phenomenon shows that the huge increase in conductance for PSS-coated NB must be a coupling effect between the polymer and $\mathrm{ZnO}$. This study shows the distinct effect of different polymers on the photoconductance of $\mathrm{ZnO} \mathrm{NB}$.

From our measurement data, coating of polymers normally increases the UV response of $\mathrm{ZnO} \mathrm{NB}$ by $10-100$ times. However, the UV response of PSS-coated NB has a much larger magnitude. To explore the reason for this distinct performance, we have measured the UV absorption spectra of the four polymers used in our study. As shown in Figure 1b, PS-co-MAc MA, PNIPAM, and CMC show a UV absorption peak at wavelengths around 190$200 \mathrm{~nm}$. In the $280 \mathrm{~nm}$ wavelength range as provided by the UV source in our experiments, the three polymers show little absorption. However, as for the UV absorption spectrum of PSS, there are two absorption peaks around 190 and $260 \mathrm{~nm}$, matching well to the excitation wavelength of the UV source. The data suggest that the large absorbance of the PSS to the UV is likely responsible for the observed huge increase in photoconductance. 

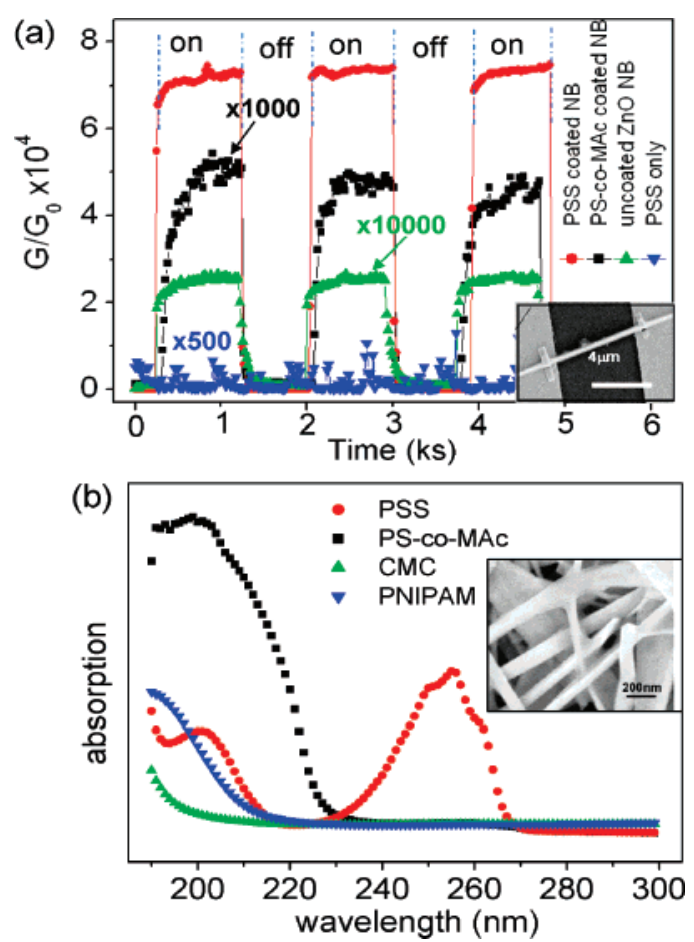

Figure 1. (a) Normalized conductance responses of a single functionalized $\mathrm{ZnO} \mathrm{NB}$ device (see inset) upon UV illumination being tuned on and off: bare $\mathrm{ZnO}$ NB without coating (green line, magnified by 10000), PS-coMAc-coated ZnO NB (dark line, magnified by 1000), PSS-coated ZnO NB (red line), PSS polymer only (blue line, magnified by 500 ). Inset is an SEM image of a typical device. The applied voltage remained at $1 \mathrm{~V}$ for all of the measurements. (b) UV absorption spectra of PSS (red line), PS-coMAc (dark line), PNIPAM (blue line), CMC (green line). Inset is an SEM image of a polymer-coated $\mathrm{ZnO} \mathrm{NB}$ sample.

Soci et al. ${ }^{9}$ have proposed that the high sensitivity UV detection of $\mathrm{ZnO} \mathrm{NW}$ was due to the presence of oxygen-related hole-trapping states at the NW surface. This process can also be applied to explain our observation. The first layer of coated polymer is cationically charged PDADMAC. The free electrons might be trapped by the positively charged sites at the surface. Upon UV illumination, these immobilized electrons can trap the photon-generated holes and thus reduce the electron-hole recombination rates in $\mathrm{ZnO} \mathrm{NB}$ and increase the carrier lifetime. This mechanism accounts for the enhanced UV response for $\mathrm{ZnO} \mathrm{NB}$ coated with PS-co-Mac, PNIPAM, and CMC.

The above mechanism may not be sufficient to account for the huge increase in photoresponse of the PSS-coated NBs. From Figure $1 \mathrm{~b}$, the UV absorption spectrum of PSS has a peak at around 260 $\mathrm{nm}$, which is coincidently close to the applied UV source wavelength. We proposed that this absorption peak and the related molecular energy states in PSS play a significant role for enhancing the photon response. Although the first layer of $\mathrm{ZnO} \mathrm{NB}$ surface is covered by PDADMAC, it is well-known that the monolayer adsorption usually cannot reach $100 \%$ coverage. Therefore, the remaining surface could be covered by the next adsorbed layer of PSS, as shown in Figure 2b. We now consider the area that PSS is directly interfacing with $\mathrm{ZnO}$. Under UV light, an electron in PSS is excited from the ground-state energy state to an exited energy state owing to high absorption, which creates an unoccupied energy state (step 1). If the ground energy state of the PSS is at the level within the band gap of $\mathrm{ZnO}$, it is possible that the electron in the valence band of $\mathrm{ZnO} \mathrm{NB}$ is likely to be excited to the ground state of PSS, which subsequently transits to the conduction band of $\mathrm{ZnO}$ (step 2). This "hopping" process may largely enhance the transition probability of the valence electrons in $\mathrm{ZnO}$ to its conduction band, resulting in a large increase of electron-hole pairs (Figure 2a).

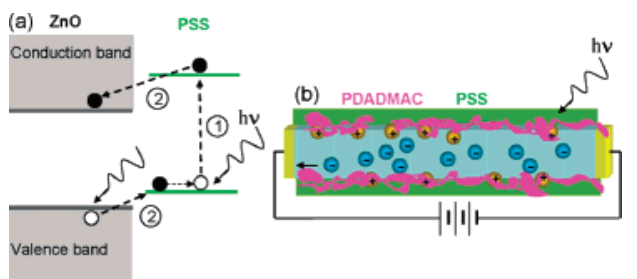

Figure 2. (a) Schematic illustration of the electron-hole generation process with facilitation of transition states from the molecular energy states in PSS. (b) Schematic illustration of photon-induced carrier transportation process in a $\mathrm{ZnO} \mathrm{NB}$ by UV illumination.

The role played by PSS is to serve as a hopping-state or bridge for the electron transfer. As discussed above, the as generated holes are trapped at the NB surface by the PDADMAC, while the electrons are transported through the NB core, (Figure 2b). Therefore, the conjunction of electron-hole pair generation in $\mathrm{ZnO}$ with the assistance of PSS and surface-hole trapping effect by PDADMAC may largely prevent the electron-hole recombination, resulting in a huge increase in the photoconductance of PSS-coated $\mathrm{ZnO}$ NB.

In conclusion, by coating $\mathrm{ZnO} \mathrm{NB}$ using a polymer that exhibits a large UV absorption peak, the UV induced photoconductance increases by 5 orders of magnitude. This huge increase in photoconductance is suggested owing to an electron-hole generation process as assisted by the energy states in the polymer. Therefore, with coating UV sensitive polymers, the UV response of $\mathrm{ZnO} \mathrm{NB}$ was greatly enhanced. This study sets the foundation for increasing the sensitivity of UV detectors based on $\mathrm{ZnO}$. The result suggests that, by selecting polymers with different wavelengths of UV absorption, UV detectors with a large range of wavelengths and super-high sensitivity can be fabricated using arrays of $\mathrm{ZnO} \mathrm{NWs}$ and NBs functionalized using different polymers. This discovery can have outstanding applications in photonics and optoelectronics.

Acknowledgment. This work was supported by DARPA STTR through Magnolia Optical Inc. and DOE BES (Grant DE-FG0207ER46394).

Supporting Information Available: UV response of PNIPAM and CMC-coated ZnO NBs. Response of PSS-coated ZnO NB to 523 and $680 \mathrm{~nm}$ wavelength. This material is available free of charge via the Internet at http://pubs.acs.org.

\section{References}

(1) Nakayama, Y.; Pauzauskie, P. J.; Radenovic, A.; Onorato, R. M.; Saykally, R. J.; Liphardt, J.; Yang, P. D. Nature 2007, 447, 1098.

(2) Pearton, S. J.; Norton, D. P.; Ip, K.; Heo, Y. W.; Steiner, T. Prog. Mater. Sci. 2005, 50, 293.

(3) Cha, S. N.; Jang, J. E.; Choi, Y.; Amaratunga, G. A. J.; Ho, G. W.; Welland, M. E.; Hasko, D. G.; Kang, D. J.; Kim, J. M. Appl. Phys. Lett. 2006, 89, 263102

(4) Lao, C. S.; Liu, J.; Gao, P. X.; Zhang, L. Y.; Davidovic, D.; Tummala, R.; Wang, Z. L. Nano Lett. 2006, 6, 263.

(5) Wang, Z. L. Adv. Mater. 2007, 19, 889.

(6) Wang, Z. L. Mater. Today 2007, 10, 20

(7) Wang, X. D.; Song, J. H.; Liu, J.; Wang, Z. L. Science 2007, 316, 102

(8) Wang, X. D.; Zhou, J.; Song, J. H.; Liu, J.; Xu, N. S.; Wang, Z. L. Nano Lett. 2006, 6, 2768.

(9) Wang, Z. L.; Song, J. H. Science 2006, 312, 242

(10) Soci, C.; Zhang, A.; Xiang, B.; Dayeh, S. A.; Aplin, D. P. R.; Park, J.; Bao, X. Y.; Lo, Y. H.; Wang, D. Nano Lett. 2007, 7, 1003.

(11) Lao, C. S.; Li, Y.; Wong, C. P.; Wang, Z. L. Nano Lett. 2007, 7, 1323.

(12) Park, W. I.; Kim, J. S.; Yi, G. C.; Bae, M. H.; Lee, H. J. Appl. Phys. Lett. 2004, 85, 5052 .

(13) Khrenov, V.; Klapper, M.; Koch, M.; Mullen, K. Macromol. Chem. Phys 2005, 206, 95

(14) Lao, C. S.; Kuang, Q.; Wang, Z. L.; Park, M. C.; Deng, Y. L. Appl. Phys. Lett. 2007, 90, 262107.

JA075249W 